\title{
Study on variable coefficients singular differential equation via constant coefficients differential equation
}

Shaowen Yao ${ }^{1}$ and Jie Liu' ${ }^{1 *}$

\section{"Correspondence:}

jzyzliujie@hpu.edu.cn

'School of Mathematics and Information Science, Henan

Polytechnic University, Jiaozuo, P.R. China

\begin{abstract}
In this paper, we consider the following third-order singular differential equation with variable coefficients:

$$
x^{\prime \prime \prime}+a(t) x=f(t, x)+e(t)
$$

By using the Green's function of the linear differential equation with constant coefficients and some fixed point theorems, i.e., Leray-Schauder alternative principle and Schauder's fixed point theorem, we prove the existence of positive periodic solutions of the above equation.
\end{abstract}

Keywords: Singular differential equations; Positive periodic solutions; Variable coefficients; Constant coefficients; Green's function

\section{Introduction}

Since the 1980s, many researchers began to discuss the singular differential equations. More concretely, in 1987, Lazer and Solimini [11] investigated the singular equations

$$
x^{\prime \prime}=-\frac{h(t)}{x^{\lambda}}+f(t)
$$

and

$$
x^{\prime \prime}=\frac{g(t)}{x^{\lambda}}+f(t)
$$

where $\lambda>0, g, h$ and $f$ are periodic functions with period $\omega$. In [11], the authors said that the (1.1) has an attractive singularity, whereas (1.2) has a repulsive singularity. For positive constant functions $h, g$ and a continuous forcing $\operatorname{term} f$, the authors gave the necessary and sufficient conditions for the existence of periodic solutions of (1.1) and (1.2). One of the common conditions to guarantee the existence of a positive periodic solution is a socalled strong force condition (corresponding to the case $\lambda \geq 1$ in (1.1)) or (1.2); see [3, $9,10,19,20,22]$ and the references therein. On the other hand, the existence of positive periodic solutions of the singular differential equations has been established with a weak

(C) The Author(s) 2019. This article is distributed under the terms of the Creative Commons Attribution 4.0 International License (http://creativecommons.org/licenses/by/4.0/), which permits unrestricted use, distribution, and reproduction in any medium, provided you give appropriate credit to the original author(s) and the source, provide a link to the Creative Commons license, and indicate if changes were made. 
force condition (corresponding to the case $0<\lambda<1$ in (1.1) or (1.2)); see [2, 4-7, 12, 13, $16,18]$.

From then on, the study of existence of positive periodic solutions for second-order singular differential equations has attracted many researchers' attention (see [2, 3, 6, 7, 9, $10,12,16,18,20])$. For example, in 2007 Torres [16] investigated a kind of second-order non-autonomous singular differential equation,

$$
x^{\prime \prime}+a(t) x=f(t, x)+e(t) .
$$

By Schauder's fixed point theorem, the author showed that the additional assumption of a weak singularity is enough to guarantee the existence of periodic solutions. Afterwards, Chu and Torres [6] improved the above results and presented a new assumption weaker than the singular condition in [16]. In 2010, Wang [18] investigated the existence and multiplicity of positive periodic solutions of the singular systems (1.3) with superlinearity or sublinearity assumptions at infinity for some $e(t)$. The proof of their results was based on the Krasnoselskii fixed point theorem.

At the same time, some authors began to consider third-order singular differential equation. For example, in 2006, Chu and Zhou [8] discussed the third-order singular differential equation

$$
x^{\prime \prime \prime}+\kappa_{*}^{3} x=f(t, x), \quad 0 \leq t \leq 2 \pi,
$$

with periodic boundary conditions $x^{(i)}(0)=x^{(i)}(2 \pi), i=0,1,2$. Here $\kappa$ is a positive constant and nonlinearity $f(t, x)$ is singular at $x=0$. By the Green's function and a fixed point theorem in cones, they obtained the existence of periodic solutions for (1.4) in a small range of $\kappa_{*}$, and to be concrete $\kappa_{*} \in\left(0, \frac{1}{\sqrt{3}}\right)$.

All the aforementioned results concern second-order singular differential equations with variable coefficients or singular third-order differential equations with constant coefficients. There are few results on the singular third-order equation with variable coefficients. Motivated by $[6,8,16,18]$, in this paper, we discuss the existence of positive periodic solutions for the following singular differential equation with variable coefficients:

$$
x^{\prime \prime \prime}+a(t) x=f(t, x)+e(t)
$$

where $e(t) \in C(\mathbb{R}, \mathbb{R})$ is an $\omega$-periodic function, $a(t) \in C\left(\mathbb{R}, \mathbb{R}^{+}\right)$is an $\omega$-periodic function and the nonlinear term $f(t, x) \in C\left(\mathbb{R} \times \mathbb{R}^{+}, \mathbb{R}\right)$. Moreover, $f$ is an $\omega$-periodic function on $t$ and is of singularity at origin, i.e.,

$$
\lim _{x \rightarrow 0^{+}} f(t, x)=+\infty, \quad\left(\text { or } \lim _{x \rightarrow 0^{+}} f(t, x)=-\infty\right), \quad \text { uniformly in } t .
$$

We say (1.5) is of repulsive type (resp. attractive type) if $f(t, x) \rightarrow+\infty$ (resp. $f(t, x) \rightarrow-\infty)$ as $x \rightarrow 0^{+}$.

As is well known, it is very complicated to calculate the Green's function of a third-order linear differential equation with variable coefficients

$$
x^{\prime \prime \prime}(t)+a(t) x(t)=h(t)
$$


where $h \in C\left(\mathbb{R}, \mathbb{R}^{+}\right)$is an $\omega$-periodic function. In this paper, we first discuss the Green's function of the third-order linear differential equation with constant coefficients

$$
x^{\prime \prime \prime}(t)+M x(t)=h(t)
$$

where $M:=\max _{t \in[0, \omega]} a(t)$. By application of the Green's function of (1.6) and some fixed point theorems, i.e. Schauder's fixed point theorem and a nonlinear alternative principle of Leray-Schauder, we obtain the existence of a positive periodic solution for (1.5).

The paper is organized as follows: In Sect. 2, the Green's function for constant coefficients differential equation (1.6) will be given. Some useful properties for the Green's function are shown also. In Sect. 3, we will prove that a weak singularity enables the achievement of new existence criteria by means of Schauder's fixed point theorem. Moreover, we consider the periodic solution of (1.5) with attractive-repulsive singularities. In Sect. 4, by employing Green's function of (1.6) and the nonlinear alternative principle of LeraySchauder, we prove the existence results of positive periodic solutions of (1.5), which are applicable to the case of a strong singularity as well as to the case of a weak singularity. Our new results generalize some recent results contained in [8].

\section{Preliminary}

\subsection{Constant coefficients differential equation}

In this section, we discuss the Green's function of the third-order differential equations

$$
\left\{\begin{array}{l}
x^{\prime \prime \prime}+M x=h(t), \\
x(0)=x(\omega), \quad x^{\prime}(0)=x^{\prime}(\omega), \quad x^{\prime \prime}(0)=x^{\prime \prime}(\omega)
\end{array}\right.
$$

where $M:=\max _{t \in[0, \omega]} a(t)$. We will use it to investigate the existence of a positive periodic solution for (1.5). In the following, we introduce Green's functions of (2.1) and some properties, which can be found in [14].

Lemma 2.1 (see [14]) The equation (2.1) has an unique $\omega$-periodic solution

$$
x(t)=\int_{0}^{\omega} G(t, s) h(s) d s,
$$

where

$$
G(t, s)=\left\{\begin{array}{c}
\frac{2 \exp \left(\frac{1}{2} M^{\frac{1}{3}}(t-s)\right)\left[\sin \left(\frac{\sqrt{3}}{2} M^{\frac{1}{3}}(t-s)-\frac{\pi}{6}\right)-\exp \left(\frac{1}{2} M^{\frac{1}{3}} \omega\right) \sin \left(\frac{\sqrt{3}}{2} M^{\frac{1}{3}}(t-s-\omega)-\frac{\pi}{6}\right)\right]}{3 M^{\frac{2}{3}}\left(1+\exp \left(M^{\frac{1}{3}} \omega\right)-2 \exp \left(\frac{1}{2} M^{\frac{1}{3}} \omega\right) \cos \left(\frac{\sqrt{3}}{2} M^{\frac{1}{3}} \omega\right)\right)} \\
+\frac{\exp \left(M^{\frac{1}{3}}(s-t)\right)}{3 M^{\frac{2}{3}}\left(1-\exp \left(-M^{\frac{1}{3}} \omega\right)\right)}, \quad 0 \leq s<t \leq \omega, \\
\frac{2 \exp \left(\frac{1}{2} M^{\frac{1}{3}}(t+\omega-s)\right)\left[\sin \left(\frac{\sqrt{3}}{2} M^{\frac{1}{3}}(t+\omega-s)-\frac{\pi}{6}\right)-\exp \left(\frac{1}{2} M^{\frac{1}{3}} \omega\right) \sin \left(\frac{\sqrt{3}}{2} M^{\frac{1}{3}}(t-s)-\frac{\pi}{6}\right)\right]}{3 M^{\frac{2}{3}}\left(1+\exp \left(M^{\frac{1}{3}} \omega\right)-2 \exp \left(\frac{1}{2} M^{\frac{1}{3}} \omega\right) \cos \left(\frac{\sqrt{3}}{2} M^{\frac{1}{3}} \omega\right)\right)} \\
\quad+\frac{\exp \left(M^{\frac{1}{3}}(s-t-\omega)\right)}{3 M^{\frac{2}{3}}\left(1-\exp \left(-M^{\frac{1}{3}} \omega\right)\right)}, \quad 0 \leq t \leq s \leq \omega .
\end{array}\right.
$$

Now we present some properties of the Green's functions for (2.1). Denote

$$
A=\frac{1}{3 M^{\frac{2}{3}}\left(\exp \left(M^{\frac{1}{3}} \omega\right)-1\right)}, \quad B=\frac{3+2 \exp \left(-\frac{M^{\frac{1}{3}} \omega}{2}\right)}{3 M^{\frac{2}{3}}\left(1-\exp \left(-\frac{M^{\frac{1}{3}} \omega}{2}\right)\right)^{2}} .
$$


Lemma 2.2 (see [14]) $\int_{0}^{\omega} G(t, s) d s=\frac{1}{M}$ and if $M<\frac{64 \pi^{3}}{81 \sqrt{3} \omega^{3}}$ holds, then $0<A \leq G(t, s) \leq B$ for all $(t, s) \in[0, \omega] \times[0, \omega]$.

On the other hand, let $M=\varrho^{3}$, then (2.1) is transformed into

$$
\left\{\begin{array}{l}
y^{\prime}(t)+\varrho y(t)=h(t) \\
y(0)=y(\omega)
\end{array}\right.
$$

and

$$
\left\{\begin{array}{l}
x^{\prime \prime}(t)-\varrho x^{\prime}(t)+\varrho^{2} x(t)=y(t) \\
x(0)=x(\omega), \quad x^{\prime}(0)=x^{\prime}(\omega)
\end{array}\right.
$$

Moreover, the solutions of (2.4) can be written as

$$
y(t)=\int_{0}^{\omega} G_{1}(t, s) h(s) d s
$$

where

$$
G_{1}(t, s)= \begin{cases}\frac{e^{-\varrho(t-s)}}{1-e^{-\omega \varrho}}, & 0 \leq s \leq t \leq \omega \\ \frac{e^{-\varrho(\omega+t-s)}}{1-e^{-\omega \varrho}}, & 0 \leq t<s \leq \omega\end{cases}
$$

Lemma 2.3 (see [15]) The boundary problem (2.5) is equivalent to the integral equation

$$
x(t)=\int_{0}^{\omega} G_{2}(t, s) y(s) d s
$$

where

$$
G_{2}(t, s)= \begin{cases}\frac{2 e^{\frac{\varrho}{2}(t-s)}\left[\sin \frac{\sqrt{3}}{2} \varrho(\omega-t+s)+e^{-\frac{\varrho \omega}{2}} \sin \frac{\sqrt{3}}{2} \varrho(t-s)\right]}{\sqrt{3} \varrho\left(e^{\frac{\varrho \omega}{2}}+e^{-\frac{\varrho \omega}{3}}-2 \cos \frac{\sqrt{3}}{2} \varrho \omega\right)}, & 0 \leq s \leq t \leq \omega, \\ \frac{2 e^{\frac{\rho}{2}(\omega+t-s)}\left[\sin \frac{\sqrt{3}}{2} \varrho(s-t)+e^{-\frac{\varrho \omega}{2}} \sin \frac{\sqrt{3}}{2} \varrho(\omega-s+t)\right]}{\sqrt{3} \varrho\left(e^{\frac{\rho \omega}{2}}+e^{-\frac{\varrho \omega}{3}}-2 \cos \frac{\sqrt{3}}{2} \varrho \omega\right)}, & 0 \leq t<s \leq \omega .\end{cases}
$$

Moreover, for $G_{2}(t, s)$, if $\varrho<\frac{2 \pi}{\sqrt{3} \omega}$, then we have the estimates

$$
0 \leq l_{1}:=\frac{2 \sin \left(\frac{\sqrt{3}}{2} \varrho \omega\right)}{\sqrt{3} \varrho\left(e^{\frac{\varrho \omega}{2}}+1\right)^{2}} \leq G_{2}(t, s) \leq \frac{2}{\sqrt{3} \sin \left(\frac{\sqrt{3}}{2} \varrho \omega\right)}:=L_{1}, \quad \forall s, t \in[0, \omega]
$$

According to the above lemmas, the solution of (2.1) can be written as

$$
\begin{aligned}
x(t) & =\int_{0}^{\omega} G_{2}(t, \tau) \int_{0}^{\omega} G_{1}(\tau, s) h(s) d s d \tau=\int_{0}^{\omega} \int_{0}^{\omega} G_{2}(t, \tau) G_{1}(\tau, s) h(s) d s d \tau \\
& =\int_{0}^{\omega}\left[\int_{0}^{\omega} G_{2}(t, s) G_{1}(s, \tau) d s\right] h(\tau) d \tau=\int_{0}^{\omega}\left[\int_{0}^{\omega} G_{2}(t, \tau) G_{1}(\tau, s) d \tau\right] h(s) d s .
\end{aligned}
$$


Thus, let

$$
G^{*}(t, s)=\int_{0}^{\omega} G_{2}(t, \tau) G_{1}(\tau, s) d \tau
$$

we can get

$$
x(t)=\int_{0}^{\omega} G^{*}(t, s) h(s) d s .
$$

Lemma 2.4 Assume that $M<\frac{8 \pi^{3}}{3 \sqrt{3} \omega^{3}}$ holds, then $G^{*}(t, s) \geq 0$ for all $(t, s) \in[0, \omega] \times[0, \omega]$.

Proof From Lemma 2.3, we know $G_{2}(t, s) \geq 0$. Since $G_{1}(t, s)>0$, from (2.7) we can see that $G^{*}(t, s) \geq 0$ for all $(t, s) \in[0, \omega] \times[0, \omega]$.

\subsection{Variable coefficients differential equation}

In this section, we consider the variable coefficients differential equations

$$
\left\{\begin{array}{l}
x^{\prime \prime \prime}+a(t) x=h(t), \\
x(0)=x(\omega), \quad x^{\prime}(0)=x^{\prime}(\omega), \quad x^{\prime \prime}(0)=x^{\prime \prime}(\omega)
\end{array}\right.
$$

where $h \in C\left(\mathbb{R}, \mathbb{R}^{+}\right)$is an $\omega$-periodic function and $a(t) \in C\left(\mathbb{R}, \mathbb{R}^{+}\right)$is the same $\omega$-periodic functions in (1.5). Obviously, the calculation of the Green's function of (2.9) is very complicated. To overcome this difficulty, we will make a shift on the linear term.

Let $X=\{\phi \in C(\mathbb{R}, \mathbb{R}): \phi(t+\omega)=\phi(t)\}$ with the maximum norm $\|\phi\|=\max _{t \in[0, \omega]}|\phi(t)|$. Obviously, $X$ is a Banach space. Denote

$$
M:=\max _{t \in[0, \omega]} a(t), \quad m:=\min _{t \in[0, \omega]} a(t),
$$

then (2.9) can be rewritten as

$$
x^{\prime \prime \prime}+M x=(M-a(t)) x+h(t) .
$$

Define the operators $T, H: X \rightarrow X$ by

$$
(T h)(t)=\int_{0}^{\omega} G(t, s) h(s) d s, \quad(H x)(t)=(M-a(t)) x .
$$

Clearly, $T, H$ are completely continuous. Furthermore, we see that $(T h)(t)>0$ if $M<$ $\frac{64 \pi^{3}}{81 \sqrt{3} \omega^{3}}$. At the same time we have $\|H\| \leq M-m$. By Lemma 2.1, the solution of (2.10) can be written in the form

$$
x(t)=(T h)(t)+(T H x)(t) .
$$

Therefore, we have

$$
\|T H\| \leq\|T\|\|H\| \leq \frac{M-m}{M}=1-\frac{m}{M}<1,
$$


using the fact $\int_{0}^{\omega} G(t, s) d s=\frac{1}{M}$. Hence the solution of (2.10) can be written as

$$
x(t)=(I-T H)^{-1}(T h)(t) .
$$

Define an operator $P: X \rightarrow X$,

$$
(P h)(t)=(I-T H)^{-1}(T h)(t)
$$

it is obvious that $x(t)=(P h)(t)$ is the unique periodic solution of (2.9) for any $h(t)$. Moveover, we have the following.

Lemma 2.5 $P$ is completely continuous and

$$
(T h)(t) \leq(P h)(t), \quad \forall t \in \mathbb{R} \quad \text { and } \quad\|P h\| \leq \frac{M}{m}\|T h\| .
$$

Proof By the Neumann expansion of $P$, we have

$$
\begin{aligned}
P & =(I-T H)^{-1} T \\
& =\left(I+T H+(T H)^{2}+\cdots+(T H)^{n}+\cdots\right) T \\
& =T+T H T+(T H)^{2} T+\cdots+(T H)^{n} T+\cdots .
\end{aligned}
$$

Since $T$ and $H$ are completely continuous, $P$ is completely continuous. Since $T h(t)>0$ for any $t$, we get

$$
(T h)(t) \leq(P h)(t) .
$$

Noting that $\|T H\|<1$, we get

$$
\|P h\| \leq \frac{M}{m}\|T h\| .
$$

For the singular differential equation with variable coefficients considered in this paper,

$$
x^{\prime \prime \prime}+a(t) x=f(t, x)+e(t)
$$

where $e(t) \in C(\mathbb{R}, \mathbb{R})$ is an $\omega$-periodic function, $a(t) \in C\left(\mathbb{R}, \mathbb{R}^{+}\right)$is an $\omega$-periodic function and the nonlinear term $f(t, x) \in C\left(\mathbb{R} \times \mathbb{R}^{+}, \mathbb{R}\right)$, we define an operator $Q: X \rightarrow X$,

$$
(Q x)(t)=P(f(t, x)+e(t)) .
$$

According to Lemma 2.5, it is easy to verify the following remark.

Remark $1 Q$ is completely continuous in $X$. 


\section{Weak singularity}

In this section, we establish the existence of positive periodic solutions for third-order differential equation (1.5) by using Schauder's fixed point theorem [21].

We write $d(t) \succ 0$ if $d(t) \geq 0$ for a.e. $t \in[0, \omega]$ and $d(t)>0$ on a set of positive measure. For a given function $e(t) \in C[0, \omega]$, we denote the essential supremum and infimum by $e^{*}$ and $e_{*}$ if they exist. Define the function $\gamma: \mathbb{R} \rightarrow \mathbb{R}$,

$$
\gamma(t)=\int_{0}^{\omega} G(t, s) e(s) d s
$$

which is the unique $\omega$-periodic solution of

$$
x^{\prime \prime \prime}(t)+M x(t)=e(t)
$$

\subsection{Case (I) $\gamma_{*}=0$}

Theorem 3.1 Suppose that $M<\frac{64 \pi^{3}}{81 \sqrt{3} \omega^{3}}$ holds. Furthermore, assume that the following conditions hold:

$\left(H_{1}\right)$ For each $L>0$, there exists a continuous function $\phi_{L} \succ 0$ such that $f(t, x) \geq \phi_{L}(t)$ for all $(t, x) \in[0, \omega] \times(0, L]$.

$\left(H_{2}\right)$ There exist continuous, non-negative functions $g(x), h(x)$ and $\zeta(t)$ such that

$$
0 \leq f(t, x) \leq \zeta(t)(g(x)+h(x)) \quad \text { for all }(t, x) \in[0, \omega] \times(0, \infty)
$$

and $g(x)>0$ is non-increasing and $h(x)$ is non-decreasing in $x \in(0, \infty)$.

$\left(H_{3}\right)$ There exists a positive constant $R>0$ such that $R>\left(\Phi_{R}\right)_{*}$ and

$$
\left.R \geq \frac{M}{m}\left(\left(g\left(\left(\Phi_{R}\right)_{*}\right)\right)\left(1+\frac{h(R)}{g(R)}\right)\right) \Lambda^{*}+\|\gamma\|\right)
$$

where

$$
\Phi_{R}(t)=\int_{0}^{\omega} G(t, s)\left(\phi_{R}\right)(s) d s, \quad \Lambda(t)=\int_{0}^{\omega} G(t, s) \zeta(s) d s, \quad\|\gamma\|=\max _{t \in[0, \omega]}|\gamma(t)| .
$$

If $\gamma_{*}=0$, then (1.5) has at least one positive periodic solution.

Proof It is obvious that an $\omega$-periodic solution of (1.5) is just a fixed point of the operator equation

$$
x(t)=(Q x)(t) .
$$

Let $R$ be the positive constant satisfying $\left(H_{3}\right)$ and denote

$$
r:=\left(\Phi_{R}\right)_{*},
$$

then we have $R>r>0$. Now define

$$
\Omega=\{x \in X: r \leq x(t) \leq R \text { for all } t\} .
$$

Obviously, $\Omega$ is a closed convex set. Next we will prove $Q(\Omega) \subset \Omega$. 
In fact, for each $x \in \Omega$ and for all $t \in[0, \omega]$, using the fact that $G(t, s)>0$ for all $(t, s) \in$ $[0, \omega] \times[0, \omega]$, together with condition $\left(H_{1}\right)$ and Lemma 2.5 , we have

$$
\begin{aligned}
(Q x)(t) & =P(f(t, x(t)+e(t)) \\
& \geq T(f(t, x(t))+e(t)) \\
& =\int_{0}^{\omega} G(t, s) f(s, x(s)) d s+\gamma(t) \\
& \geq \int_{0}^{\omega} G(t, s) \phi_{R}(s) d s+\gamma(t) \\
& \geq\left(\Phi_{R}\right)_{*}:=r>0 .
\end{aligned}
$$

On the other hand, by conditions $\left(H_{2}\right),\left(H_{3}\right)$ and Lemma 2.5 , we have

$$
\begin{aligned}
(Q x)(t) & =P(f(t, x(t))+e(t)) \\
& \leq \frac{M}{m} \max _{t \in[0, \omega]}|T(f(t, x(t))+e(t))| \\
& =\frac{M}{m} \max _{t \in[0, \omega]}\left|\int_{0}^{\omega} G(t, s)(f(s, x(s))+e(s)) d s\right| \\
& =\frac{M}{m} \max _{t \in[0, \omega]}\left|\int_{0}^{\omega} G(t, s) f(s, x(s)) d s+\gamma(t)\right| \\
& \leq \frac{M}{m}\left\{\max _{t \in[0, \omega]}\left|\int_{0}^{\omega} G(t, s) f(s, x(s)) d s\right|+\|\gamma\|\right\} \\
& \leq \frac{M}{m} \max _{t \in[0, \omega]}\left|\int_{0}^{\omega} G(t, s) \zeta(t)(g(x(s))+h(x(s))) d s\right|+\frac{M}{m}\|\gamma\| \\
& \leq \frac{M}{m}\left(g(r)\left(1+\frac{h(R)}{g(R)}\right) \Lambda^{*}+\|\gamma\|\right) \leq R .
\end{aligned}
$$

In conclusion, $Q(\Omega) \subset \Omega$. From Remark 1, we know that $Q$ is compact in $\Omega$. Therefore, the proof is completed by Schauder's fixed point theorem.

Corollary 3.2 Suppose that $M<\frac{64 \pi^{3}}{81 \sqrt{3} \omega^{3}}$ holds. Assume the following condition holds:

$\left(F_{1}\right)$ There exist continuous functions $d(t), \hat{d}(t) \succ 0$ and $0<\rho<1$ such that satisfy

$$
0 \leq \frac{\hat{d}(t)}{x^{\rho}} \leq f(t, x) \leq \frac{d(t)}{x^{\rho}}, \text { for all } x>0 \text {, and a.e.t. }
$$

If $\gamma_{*}=0$, then (1.5) has at least one positive periodic solution.

Proof Take

$$
\phi_{L}(t)=\frac{\hat{d}(t)}{L^{\rho}}, \quad \zeta(t)=d(t), \quad g(x)=\frac{1}{x^{\rho}}, \quad h(x)=0,
$$

then conditions $\left(H_{1}\right)$ and $\left(H_{2}\right)$ are satisfied and the existence condition $\left(H_{3}\right)$ becomes

$$
R>\frac{\hat{\Psi}_{*}}{R^{\rho}}=r, \quad \frac{M}{m}\left(\left(\frac{R^{\rho}}{\hat{\Psi}_{*}}\right)^{\rho} \Psi^{*}+\|\gamma\|\right) \leq R,
$$


where $\hat{\Psi}=\int_{0}^{\omega} G(t, s) \hat{d}(s) d s, \Psi=\int_{0}^{\omega} G(t, s) d(s) d s$, for some $R>0$. Note that $\hat{\Psi}_{*}>0$, since $0<\rho<1$, we can choose $R>0$ large enough so that (3.3) is satisfied. By Theorem 3.1, we can complete the proof.

\subsection{Case (II) $\gamma_{*}>0$}

Theorem 3.3 Suppose that $M<\frac{64 \pi^{3}}{81 \sqrt{3} \omega^{3}}$ holds. And $f(t, x)$ satisfies $\left(H_{2}\right)$. Furthermore, assume that the following condition holds:

$\left(H_{4}\right)$ There exists $R>0$ such that

$$
\frac{M}{m}\left(g\left(\gamma_{*}\right)\left(1+\frac{h(R)}{g(R)}\right) \Lambda^{*}+\gamma^{*}\right) \leq R
$$

If $\gamma_{*}>0$, then (1.5) has at least one positive periodic solution.

Proof We shall adopt the same strategy and notation as in the proof of Theorem 3.1. Let $R$ be the positive constant satisfying $\left(H_{4}\right)$ and $r=\gamma_{*}$, then $R>r>0$ since $R>\gamma^{*}$. Next we prove that $Q(\Omega) \subset \Omega$.

For each $x \in \Omega$ and for all $t \in[0, \omega]$, by the non-negative sign of $G(t, s)$ and $f(t, x)$ we have

$$
\begin{aligned}
(Q x)(t) & =P(f(t, x(t))+e(t)) \\
& \geq T(f(t, x(t))+e(t)) \\
& =\int_{0}^{\omega} G(t, s) f(s, x(s)) d s+\gamma(t) \\
& \geq \gamma_{*}=r>0 .
\end{aligned}
$$

On the other hand, from Lemma 2.5, we have

$$
\begin{aligned}
(Q x)(t) & =P(f(t, x(t))+e(t)) \\
& \leq \frac{M}{m} \max _{t \in[0, \omega]}\left|\int_{0}^{\omega} G(t, s) f(s, x(s)) d s+\gamma(t)\right| \\
& \leq \frac{M}{m}\left\{\max _{t \in[0, \omega]}\left|\int_{0}^{\omega} G(t, s) f(s, x(s)) d s\right|+\gamma^{*}\right\},
\end{aligned}
$$

since $\gamma_{*}>0$, we know $\gamma(t)>0$, then $\|\gamma\|=\gamma^{*}$. Therefore, by $\left(H_{2}\right)$ and $\left(H_{4}\right)$, we have

$$
\begin{aligned}
(Q x)(t) & \leq \frac{M}{m}\left\{\max _{t \in[0, \omega]}\left|\int_{0}^{\omega} G(t, s) f(s, x(s)) d s\right|+\gamma^{*}\right\} \\
& \leq \frac{M}{m}\left\{\max _{t \in[0, \omega]}\left|\int_{0}^{\omega} G(t, s) \zeta(s)(g(x(s))+h(x(s))) d s\right|+\gamma^{*}\right\} \\
& \leq \frac{M}{m}\left(g(r)\left(1+\frac{h(R)}{g(R)}\right) \Lambda^{*}+\gamma^{*}\right) \leq R .
\end{aligned}
$$

In conclusion, $Q(\Omega) \subset \Omega$. From Remark 1, it is easy to show that $Q$ is compact in $\Omega$. Therefore, by Schauder's fixed point theorem, our result is proven. 
Corollary 3.4 Suppose that $M<\frac{64 \pi^{3}}{81 \sqrt{3} \omega^{3}}$ holds. Assume the following condition holds:

$\left(F_{2}\right)$ There exist a continuous function $d(t) \succ 0$ and a constant $\rho>0$ such that satisfy

$$
0 \leq f(t, x) \leq \frac{d(t)}{x^{\rho}}, \text { for all } x>0, \text { and a.e. } t
$$

If $\gamma_{*}>0$, then (1.5) has at least one positive periodic solution.

Proof Take

$$
\zeta(t)=d(t), \quad g(x)=\frac{1}{x^{\rho}}, \quad h(x)=0,
$$

then condition $\left(H_{2}\right)$ is satisfied and the existence condition $\left(H_{4}\right)$ is also satisfied if we take $R>0$ with

$$
R \geq \frac{M}{m}\left(\frac{\Psi^{*}}{\left(\gamma_{*}\right)^{\rho}}+\gamma^{*}\right)
$$

By Theorem 3.3, the result is obvious.

Corollary 3.5 Suppose that $M<\frac{64 \pi^{3}}{81 \sqrt{3} \omega^{3}}$ holds. Assume the following condition holds: $\left(F_{3}\right)$ There exist a continuous function $d(t) \succ 0$ and constants $\rho>0,0 \leq \eta<1$ that satisfy

$$
0 \leq f(t, x) \leq \frac{d(t)}{x^{\rho}}+d(t) x^{\eta}, \quad \text { for all } x>0, \text { and a.e. } t
$$

If $\gamma_{*}>0$, then (1.5) has at least one positive periodic solution.

Proof Take

$$
\zeta(t)=d(t), \quad g(x)=\frac{1}{x^{\rho}}, \quad h(x)=x^{\eta},
$$

then condition $\left(H_{2}\right)$ is satisfied and the existence condition $\left(H_{4}\right)$ is also satisfied if we take $R>0$ with

$$
R \geq \frac{M}{m}\left(\Psi^{*}\left(\frac{1}{\left(\gamma_{*}\right)^{\rho}}+(R)^{\eta}\right)+\gamma^{*}\right) .
$$

Using Theorem 3.3, the proof is complete.

On the other hand, condition $\left(H_{2}\right)$ implies that the nonlinearity $f(t, x)$ is non-negative for all values $(t, x)$, which is quite a hard restriction. In the following, we will show how to avoid this restriction for $\gamma_{*}>0$.

Theorem 3.6 Assume that $M<\frac{64 \pi^{3}}{81 \sqrt{3} \omega^{3}}$ holds. Furthermore, assume that the following conditions hold:

$\left(H_{2}^{\prime}\right)$ There exist continuous, non-negative functions $g(x)$ and $\zeta(t)$, such that

$$
f(t, x) \leq \zeta(t) g(x) \quad \text { for all }(t, x) \in[0, \omega] \times(0, \infty)
$$

and $g(x)>0$ is non-increasing in $x \in(0, \infty)$. 
$\left(H_{3}^{\prime}\right)$ Let us define

$$
R:=\frac{M}{m}\left(g\left(\gamma_{*}\right) \Lambda^{*}+\gamma^{*}\right)
$$

and assume that $f(t, x) \geq 0$ for all $(t, x) \in[0, \omega] \times(0, R]$.

If $\gamma_{*}>0$, then (1.5) has at least one positive periodic solution.

Proof Let $R$ be the positive constant satisfying $\left(H_{3}^{\prime}\right)$ and $r=\gamma_{*}$, then $R>r>0$ since $R>\gamma^{*}$. Using the same method in the proof of Theorem 3.3, it is easy to prove that $T(\Omega) \subset \Omega$. We omit it. Then, by Schauder's fixed point theorem, we complete the proof.

Corollary 3.7 Assume $M<\frac{64 \pi^{3}}{81 \sqrt{3} \omega^{3}}$ holds. Assume the following condition hold:

$\left(F_{4}\right)$ there exist constants $\rho, \eta, \mu>0$ such that satisfy

$$
f(t, x)=\frac{1}{x^{\rho}}-\mu x^{\eta}, \quad \text { for all } x>0 \text {, and a.e.t. }
$$

If $\gamma_{*}>0$, then (1.5) has at least one positive periodic solution for each $0 \leq \mu<\mu^{\prime}$, where $\mu^{\prime}$ is some positive constant.

Proof The nonlinearity is $f(t, x)=\frac{1}{x^{\rho}}-\mu x^{\eta}$, and therefore $\left(H_{2}^{\prime}\right)$ holds with $\zeta(t)=1, g(x)=$ $\frac{1}{x^{\rho}}$. Define $R=\frac{M}{m}\left(\frac{\gamma^{*}}{\left(\gamma_{*}\right)^{\rho}}+\gamma^{*}\right)$. Note that $f(t, x) \geq 0$ if and only if $x^{\rho+\eta} \leq 1 / \mu$. Therefore, $\left(H_{3}^{\prime}\right)$ is verified for any $\mu<(R)^{-(\rho+\eta)}$. As a consequence, the result holds for $\mu^{\prime}=\left(\frac{M}{m}\left(\frac{\gamma^{*}}{\left(\gamma_{*}\right)^{\rho}}+\right.\right.$ $\left.\left.\gamma^{*}\right)\right)^{-(\rho+\eta)}$.

In the following, we will investigate (1.5) with attractive-repulsive singularities.

Corollary 3.8 Assume that $\frac{3 \sqrt{3} \omega M^{\frac{1}{3}}}{4 \pi}<1$ holds and the nonlinearity in (1.5) is

$$
f(t, x)=\frac{1}{x^{\alpha}}-\frac{\kappa}{x^{\beta}}
$$

where $\alpha>\beta>0$ and $\kappa>0$ is a positive parameter. If $\gamma_{*}>0$ and $\kappa \in\left[0, \kappa_{1}\right]$, where

$$
\kappa_{1}=\left(\frac{M}{m}\left(\frac{1}{M\left(\gamma_{*}\right)^{\alpha}}+\gamma^{*}\right)\right)^{\beta-\alpha}
$$

then (1.5) has at least one positive periodic solution.

Proof To apply Theorem 3.6, we take

$$
g(x)=\frac{1}{x^{\alpha}}, \quad \zeta(t) \equiv 1
$$

It is obvious that the condition $\left(H_{2}\right)^{\prime}$ holds. Take $R>0$ with

$$
R=\frac{M}{m}\left(\frac{1}{M\left(\gamma_{*}\right)^{\alpha}}+\gamma^{*}\right)
$$

where $\frac{1}{M}=\int_{0}^{\omega} G(t, s) d s$. 
Next, we prove $f(t, x) \geq 0$ for all $(t, x) \in[0, \omega] \times(0, R]$. In fact, since $\alpha>\beta>0$ and $\kappa \in$ $\left[0, \kappa_{1}\right]$, we have

$$
\kappa \leq\left(\frac{M}{m}\left(\frac{1}{M\left(\gamma_{*}\right)^{\alpha}}+\gamma^{*}\right)\right)^{\beta-\alpha}=R^{\beta-\alpha} \leq x^{\beta-\alpha} \quad \forall x \in(0, R]
$$

Therefore, we get $f(t, x) \geq 0$ for all $(t, x) \in[0, \omega] \times(0, R]$. Then condition $\left(H_{3}^{\prime}\right)$ is satisfied.

\subsection{Case (III) $\gamma^{*}<0$}

Theorem 3.9 Assume that $M<\frac{64 \pi^{3}}{81 \sqrt{3} \omega^{3}}$ holds and $f(t, x)$ satisfies $\left(H_{1}\right)$ and $\left(H_{2}\right)$. Furthermore, assume that the following condition holds:

$\left(H_{5}\right)$ There exists $R>0$ such that $R>\left(\Phi_{R}\right)_{*}+\gamma_{*}>0$ and

$$
\frac{M}{m} g\left(\left(\Phi_{R}\right)_{*}+\gamma_{*}\right)\left(1+\frac{h(R)}{g(R)}\right) \Lambda^{*} \leq R
$$

If $\gamma^{*}<0$, then (1.5) has at least one positive periodic solution.

Proof This theorem can be proved in the same way as Theorem 3.1.

Let $R$ be a positive constant satisfying $\left(H_{5}\right)$ and $r=\left(\Phi_{R}\right)_{*}+\gamma_{*}$, then $R>r>0$ since $R>$ $\left(\Phi_{R}\right)_{*}+\gamma_{*}$. Next we will prove that $Q(\Omega) \subset \Omega$.

For each $x \in \Omega$ and for all $t \in[0, \omega]$, from $\left(H_{1}\right),\left(H_{5}\right)$ and Lemma 2.5 , we have

$$
\begin{aligned}
(Q x)(t) & =P(f(t, x(t))+e(t)) \geq T(f(t, x(t))+e(t)) \\
& =\int_{0}^{\omega} G(t, s) f(s, x(s)) d s+\gamma(t) \geq \int_{0}^{\omega} G(t, s) \phi_{R}(s) d s+\gamma(t) \\
& \geq\left(\Phi_{R}\right)_{*}+\gamma_{*}=r>0 .
\end{aligned}
$$

On the other hand, from Lemma 2.5, we have

$$
\begin{aligned}
(Q x)(t) & =P(f(t, x(t))+e(t)) \leq \frac{M}{m} \max _{t \in[0, \omega]}|T(f(t, x(t))+e(t))| \\
& =\frac{M}{m} \max _{t \in[0, \omega]}\left|\int_{0}^{\omega} G(t, s) f(s, x(s)) d s+\gamma(t)\right| \\
& \leq \frac{M}{m} \max _{t \in[0, \omega]}\left|\int_{0}^{\omega} G(t, s) f(s, x(s)) d s\right|,
\end{aligned}
$$

since $\gamma^{*} \leq 0, G(t, s)$ and $f(t, x(t))$ are non-negative, $\left(\Phi_{R}\right)_{*}+\gamma_{*}>0$, then we know

$$
\left|\int_{0}^{\omega} G(t, s) f(s, x(s)) d s+\gamma(t)\right| \leq\left|\int_{0}^{\omega} G(t, s) f(s, x(s)) d s\right| .
$$


Therefore, by $\left(H_{2}\right)$ and $\left(H_{5}\right)$, we have

$$
\begin{aligned}
(Q x)(t) & \leq \frac{M}{m}\left\{\max _{t \in[0, \omega]}\left|\int_{0}^{\omega} G(t, s) f^{+}(s, x(s)) d s\right|\right\} \\
& \leq \frac{M}{m}\left\{\max _{t \in[0, \omega]}\left|\int_{0}^{\omega} G(t, s) \zeta(s)(g(x(s))+h(x(s))) d s\right|\right\} \\
& \leq \frac{M}{m} g(r)\left(1+\frac{h(R)}{g(R)}\right) \Lambda^{*} \leq R .
\end{aligned}
$$

In conclusion, $Q(\Omega) \subset \Omega$. From Remark 1 , it is easy to verify that $Q$ is compact in $\Omega$. Therefore, by Schauder's fixed point theorem, our result is proven.

Corollary 3.10 Assume $M<\frac{64 \pi^{3}}{81 \sqrt{3} \omega^{3}}$ and $\left(F_{1}\right)$ hold. If $\gamma^{*}<0$ and

$$
\gamma_{*} \geq\left(\frac{\hat{\Psi}_{*} m^{\rho}}{\left(M \Psi^{*}\right)^{\rho}} \rho^{2}\right)^{\frac{1}{1-\rho^{2}}}\left(1-\frac{1}{\rho^{2}}\right)
$$

where $0<\rho<1$ appears in $\left(F_{1}\right)$, then there exists a positive periodic solution of $(1.5)$.

Proof Take

$$
\phi_{L}(t)=\frac{\hat{d}(t)}{L^{\rho}}, \quad \zeta(t)=d(t), \quad g(x)=\frac{1}{x^{\rho}}, \quad h(x)=0,
$$

Then conditions $\left(H_{1}\right)$ and $\left(H_{2}\right)$ are satisfied. Next, we consider the condition $\left(H_{5}\right)$ also to be satisfied. Taking $R:=\frac{M \Psi^{*}}{m(r)^{\rho}}$, then $\left(\Phi_{R}\right)_{*}+\gamma_{*}>0$ holds if $r$ satisfies

$$
\frac{\hat{\Psi}_{*} m^{\rho}}{\left(M \Psi^{*}\right)^{\rho}}(r)^{\rho^{2}}+\gamma_{*} \geq r
$$

or, equivalently,

$$
\gamma_{*} \geq f(r):=r-\frac{\hat{\Psi}_{*} m^{\rho}}{\left(M \Psi^{*}\right)^{\rho}}(r)^{\rho^{2}}
$$

The function $f(r)$ possesses a minimum at $r_{0}:=\left[\frac{\hat{\Psi}_{*} m^{\rho}}{\left(M \Psi^{*}\right)^{\rho}} \rho^{2}\right]^{\frac{1}{1-\rho^{2}}}$. Let $r=r_{0}$, then $\left(\Phi_{R}\right)_{*}+\gamma_{*}>$ 0 holds in $\left(H_{5}\right)$ if $\gamma_{*} \geq f\left(r_{0}\right)$, which is just the condition

$$
\gamma_{*} \geq\left(\frac{\hat{\Psi}_{*} m^{\rho}}{\left(M \Psi^{*}\right)^{\rho}} \rho^{2}\right)^{\frac{1}{1-\rho^{2}}}\left(1-\frac{1}{\rho^{2}}\right)
$$

The $\left(H_{5}\right)$ holds directly by the choice of $R$, and it remain to prove that $R=\frac{M \Psi^{*}}{m\left(r_{0}\right)^{\rho}}>r_{0}$. This is easily verified through elementary computations. Using Theorem 3.9, we complete the proof.

\section{Strong and weak singularities}

In the section, we state and prove the existence results which are applicable to the case of a strong singularity as well as to the case of a weak singularity. The proof is based on the following nonlinear alternative of Leray-Schauder, which can be found in [1]. 
Lemma 4.1 ([1]) Let $C$ be a convex subset of a normed linear space $E$, and let $U$ be an open subset of $C$ with $0 \in U$. Then every compact, continuous map $F: \bar{U} \rightarrow C$ has at least one of the following properties,

(I) F has a fixed point in $\bar{U}$; or

(II) there is $a x \in \partial U$ and $\lambda \in(0,1)$ with $x=\lambda F x$.

Let

$$
A=\min _{0 \leq s, t \leq \omega} G(t, s), \quad B=\max _{0 \leq s, t \leq \omega} G(t, s), \quad \sigma=A / B .
$$

By Lemma 2.2, we have $B>A>0$ and $0<\sigma<1$.

Define the operator $Q^{*}: X \rightarrow X$,

$$
\left(Q^{*} x\right)(t)=P(f(t, x(t)+\gamma(t)))
$$

and a cone $K$ in $X$,

$$
K=\left\{x \in X: x(t) \geq 0 \text { for all } t \in R \text { and } \min _{t \in R} x(t) \geq \frac{\sigma m}{M}\|x\|\right\} .
$$

\subsection{Case (I) $\gamma_{*} \geq 0$}

Theorem 4.2 Assume that $M<\frac{64 \pi^{3}}{81 \sqrt{3} \omega^{3}},\left(H_{1}\right)$ and $\left(H_{2}\right)$ hold. Suppose the following conditions are satisfied:

$\left(H_{6}\right)$ there exists a positive constant $R>0$ such that

$$
\frac{M}{m} g\left(\frac{\sigma m}{M} R+\gamma_{*}\right)\left(1+\frac{h\left(R+\gamma^{*}\right)}{g\left(R+\gamma^{*}\right)}\right) \Lambda^{*}<R
$$

If $\gamma_{*} \geq 0$, then (1.5) has at least one positive $\omega$-periodic solution $x$ with $x(t)>\gamma(t)$ for all $t$ and $0<\|x-\gamma\|<R$.

Proof Step 1. Consider the equation

$$
x^{\prime \prime \prime}(t)+a(t) x(t)=f(t, x(t)+\gamma(t))
$$

It is easy to see that if (4.2) has a positive $\omega$-periodic solution $x$ satisfying $x(t)+\gamma(t)>0$ for $t \in[0, \omega]$ and $0<\|x\|<R$, then $u(t)=x(t)+\gamma(t)$ is a positive $\omega$-periodic solution of (1.5) with $0<\|u-\gamma\|<R$. So we only need to consider (4.2).

Since $\left(H_{6}\right)$ holds, we can choose $n_{0} \in\{1,2, \ldots\}$ such that $\frac{1}{n_{0}}<\frac{\sigma m}{M} R+\gamma_{*}$ and

$$
\frac{M}{m}\left(g\left(\frac{\sigma m}{M} R+\gamma_{*}\right)\left(1+\frac{h\left(R+\gamma^{*}\right)}{g\left(R+\gamma^{*}\right)}\right) \Lambda^{*}+\frac{1}{n_{0}}\right)<R
$$

Let $N_{0}=\left\{n_{0}, n_{0}+1, \ldots\right\}$ and fix $n \in N_{0}$, we consider the family of equations

$$
x^{\prime \prime \prime}(t)+a(t) x(t)=\mu f_{n}(t, x(t)+\gamma(t))+\frac{M}{n},
$$


where $\mu \in[0,1]$ and

$$
f_{n}(t, x)= \begin{cases}f(t, x) & \text { if } x \geq \frac{1}{n} \\ f\left(t, \frac{1}{n}\right) & \text { if } x \leq \frac{1}{n}\end{cases}
$$

Now we prove that (4.3) has a periodic solution for each $n$. If $x$ is a periodic solution of problem (4.3), define an operator $Q_{n}^{*}: K \rightarrow X$ by

$$
\left(Q_{n}^{*} x\right)(t)=P\left(\mu f_{n}(t, x(t)+\gamma(t))+\frac{M}{n}\right)
$$

So, solving (4.3) is equivalent to solving the following operator equation:

$$
x(t)=\left(Q_{n}^{*} x\right)(t) .
$$

Let $\Omega=\{x \in K \mid\|x\|<R\}$. We claim $Q_{n}^{*}(\Omega) \subset K$. In fact, $\forall x \in K$, from Lemma 2.5, we have

$$
\begin{aligned}
\min _{t \in \mathbb{R}}\left(Q_{n}^{*} x\right)(t) & =\min _{t \in \mathbb{R}} P\left(\mu f_{n}(t, x(t)+\gamma(t))+\frac{M}{n}\right) \\
& \geq \min _{t \in \mathbb{R}} T\left(\mu f_{n}(t, x(t)+\gamma(t))+\frac{M}{n}\right) \\
& =\mu \min _{t \in \mathbb{R}} \int_{0}^{\omega} G(t, s) f_{n}(s, x(s)+\gamma(s)) d s+\frac{1}{n},
\end{aligned}
$$

since $\int_{0}^{\omega} G(t, s)=\frac{1}{M}$. Therefore, from Lemma 2.2 and Lemma 2.5, we have

$$
\begin{aligned}
\min _{t \in \mathbb{R}}\left(Q_{n}^{*} x\right)(t) & \geq \mu A \int_{0}^{\omega} f_{n}(s, x(s)+\gamma(s)) d s+\frac{1}{n} \\
& =\mu \sigma B \int_{0}^{\omega} f_{n}(s, x(s)+\gamma(s)) d s+\frac{1}{n} \\
& \geq \sigma \max _{t \in \mathbb{R}} \mu \int_{0}^{\omega} G(t, s) f_{n}(s, x(s)+\gamma(s)) d s+\frac{1}{n} \\
& =\sigma \max _{t \in \mathbb{R}} T\left(\mu f_{n}(t, x(t)+\gamma(t))+\frac{M}{n}\right) \\
& \geq \sigma \frac{m}{M} \max _{t \in \mathbb{R}} P\left(\mu f_{n}(t, x(t)+\gamma(t))+\frac{M}{n}\right) \\
& =\sigma \frac{m}{M}\left\|Q_{n}^{*} x\right\| .
\end{aligned}
$$

Since $(T h)(t) \leq(P h)(t) \leq \frac{M}{m}\|T h\|$, we can get $\|T h\| \geq \frac{m}{M}\|P h\|$. This implies that $Q_{n}^{*}(\Omega) \subset K$. Besides, from Lemma 2.5 , we know that $Q_{n}^{*}: \Omega \rightarrow K$ is completely continuous.

Step 2. We claim that any fixed point $x$ of (4.4) for all $\mu \in[0,1]$ must satisfy $\|x\| \neq R$. Otherwise, assume that $x$ is a fixed point of (4.4) for some $\mu \in[0,1]$ such that $\|x\|=R$. 
Thus, from Lemma 2.5, we have

$$
\begin{aligned}
x(t) & =\left(Q_{n}^{*} x\right)(t) \\
& =P\left(\mu f_{n}(t, x(t)+\gamma(t))+\frac{M}{n}\right) \\
& \geq T\left(\mu f_{n}(t, x(t)+\gamma(t))+\frac{M}{n}\right) \\
& =\mu \int_{0}^{\omega} G(t, s) f_{n}(s, x(s)+\gamma(s)) d s+\frac{1}{n} \\
& \geq \mu A \int_{0}^{\omega} f_{n}(s, x(s)+\gamma(s)) d s+\frac{1}{n} \\
& =\mu \sigma B \int_{0}^{\omega} f_{n}(s, x(s)+\gamma(s)) d s+\frac{1}{n} \\
& \geq \sigma \max _{t \in \mathbb{R}} \mu \int_{0}^{\omega} G(t, s) f_{n}(s, x(s)+\gamma(s)) d s+\frac{1}{n} \\
& =\sigma \max _{t \in \mathbb{R}} \int_{0}^{\omega} G(t, s)\left(\mu f_{n}(s, x(s)+\gamma(s))+\frac{M}{n}\right) d s \\
& =\sigma \max _{t \in \mathbb{R}} T\left(\mu f_{n}(t, x(t)+\gamma(t))+\frac{M}{n}\right) \\
& \geq \sigma \frac{m}{M} \max _{t \in \mathbb{R}} P\left(\mu f_{n}(t, x(t)+\gamma(t))+\frac{M}{n}\right) \\
& m{ }^{m} \| .
\end{aligned}
$$

Therefore, we have

$$
x(t)+\gamma(t) \geq \sigma \frac{m}{M}\|x\|+\gamma_{*} \geq \sigma \frac{m}{M} R+\gamma_{*}>\frac{1}{n},
$$

since $\frac{1}{n} \leq \frac{1}{n_{0}}<\sigma \frac{m}{M} R+\gamma_{*}$. Thus, from $\left(H_{2}\right)$ we have

$$
\begin{aligned}
x(t) & =P\left(\mu f_{n}(t, x(t)+\gamma(t))+\frac{M}{n}\right) \\
& \leq \frac{M}{m} \max _{t \in \mathbb{R}} T\left(\mu f_{n}(t, x(t)+\gamma(t))+\frac{M}{n}\right) \\
& \leq \frac{M}{m} \max _{t \in \mathbb{R}}\left(\mu \int_{0}^{\omega} G(t, s) f_{n}(s, x(s)+\gamma(s)) d s+\frac{1}{n}\right) \\
& =\frac{M}{m} \max _{t \in \mathbb{R}}\left(\mu \int_{0}^{\omega} G(t, s) f(s, x(s)+\gamma(s)) d s+\frac{1}{n}\right) \\
& \leq \frac{M}{m} \max _{t \in \mathbb{R}}\left(\int_{0}^{\omega} G(t, s) \zeta(s)(g(x(s)+\gamma(s)))\left(1+\frac{h(x(s)+\gamma(s))}{g(x(s)+\gamma(s))}\right) d s+\frac{1}{n}\right) \\
& \leq \frac{M}{m}\left(g\left(\sigma \frac{m}{M} R+\gamma_{*}\right)\left(1+\frac{h\left(R+\gamma^{*}\right)}{g\left(R+\gamma^{*}\right)}\right) \Lambda^{*}+\frac{1}{n_{0}}\right) .
\end{aligned}
$$


Therefore, we have

$$
R=\|x\| \leq \frac{M}{m}\left(g\left(\sigma \frac{m}{M} R+\gamma_{*}\right)\left(1+\frac{h\left(R+\gamma^{*}\right)}{g\left(R+\gamma^{*}\right)}\right) \Lambda^{*}+\frac{1}{n_{0}}\right) .
$$

This is a contradiction to the choice of $n_{0}$ and the claim is proved.

From this claim, Lemma 4.1 guarantees that

$$
x(t)=\left(Q_{n}^{*} x\right)(t)
$$

has a fixed point, denoted by $x_{n}$, in $\Omega$, i.e.,

$$
x^{\prime \prime \prime}(t)+a(t) x(t)=f_{n}(t, x(t)+\gamma(t))+\frac{M}{n}
$$

has a $\omega$-periodic solution $x_{n}$ with $\left\|x_{n}\right\|<R$. Since $x_{n}(t) \geq \frac{1}{n}>0$ for all $t \in[0, \omega], x_{n}$ is actually a positive $\omega$-periodic solution of (4.6).

Now we show that $x_{n}(t)+\gamma(t)$ have an uniform positive lower bound, i.e., there exists a constant $\vartheta>0$ independent of $n \in N_{0}$ such that

$$
\min _{t \in[0, \omega]}\left\{x_{n}(t)+\gamma(t)\right\} \geq \vartheta
$$

for all $n \in N_{0}$. To see this, we know from $\left(H_{1}\right)$ that there exists a continuous function $\phi_{R+\gamma^{*}}(t)>0$ such that $f(t, x) \geq \phi_{R+\gamma^{*}}(t)$ for a.e. $t$ and $0<x \leq R+\gamma^{*}$. Let $x_{R+\gamma^{*}}(t)$ be the unique $\omega$-periodic solution to

$$
x^{\prime \prime \prime}(t)+a(t) x(t)=\phi_{R+\gamma^{*}}(t)
$$

according to Lemma 2.5 and $\gamma_{*} \geq 0$, we have

$$
\begin{aligned}
x_{R+\gamma^{*}}(t)+\gamma(t) & =P\left(\phi_{R+\gamma^{*}}(t)\right)+\gamma(t) \geq T\left(\phi_{R+\gamma^{*}}(t)\right)+\gamma(t) \\
& =\int_{0}^{\omega} G(t, s) \phi_{r+\gamma^{*}}(s) d s+\gamma(t) \geq \Phi_{*}+\gamma_{*}>0,
\end{aligned}
$$

where $\Phi(t)=\int_{0}^{\omega} G(t, s) \phi_{r+\gamma^{*}}(s) d s$. Since $x_{n}(t)+\gamma(t) \leq R+\gamma^{*}$ and $x_{n}+\gamma_{*} \geq \frac{1}{n}$, we have

$$
\begin{aligned}
x_{n}(t)+\gamma(t) & =P\left(f_{n}\left(s, x_{n}(s)+\gamma(s)\right)+\frac{M}{n}\right)+\gamma(t) \\
& \geq T\left(f_{n}\left(s, x_{n}(s)+\gamma(s)\right)+\frac{M}{n}\right)+\gamma(t) \\
& =\int_{0}^{\omega} G(t, s) f_{n}\left(s, x_{n}(s)+\gamma(s)\right) d s+\gamma(t)+\frac{1}{n} \\
& =\int_{0}^{\omega} G(t, s) f\left(s, x_{n}(s)+\gamma(s)\right) d s+\gamma(t)+\frac{1}{n} \\
& \geq \int_{0}^{\omega} G(t, s) \phi_{R+\gamma^{*}}(s) d s+\gamma(t) \\
& \geq \Phi_{*}+\gamma_{*}:=\vartheta .
\end{aligned}
$$

So we have $x_{n}(t)+\gamma(t) \geq \vartheta$ for all $n$. 
Step 3. In order to pass from the solution $x_{n}$ of (4.6) to that of the original problem (4.2), we need to show $\left\{x_{n}\right\}_{n \in N_{0}}$ is compact.

First we claim

$$
\left\|x_{n}^{\prime}\right\| \leq D_{1}
$$

for some constant $D_{1}>0$ (independent of $n \in N_{0}$ ) and for all $n \in N_{0}$.

In fact, since $x_{n}$ is an $\omega$-periodic solution of (4.6) we have

$$
x_{n}^{\prime \prime \prime}(t)+a(t) x_{n}(t)=f_{n}\left(t, x_{n}(t)+\gamma(t)\right)+\frac{M}{n} .
$$

Multiplying both sides of (4.9) by $x_{n}^{\prime}(t)$ and integrating from 0 to $\omega$, we have

$$
\int_{0}^{\omega} x_{n}^{\prime \prime \prime}(t) x_{n}^{\prime}(t) d t+\int_{0}^{\omega} a(t) x_{n}(t) x_{n}^{\prime}(t) d t=\int_{0}^{\omega} f_{n}\left(t, x_{n}(t)+\gamma(t)\right) x_{n}^{\prime}(t) d t .
$$

Substituting $\int_{0}^{\omega} x_{n}^{\prime \prime \prime}(t) x_{n}^{\prime}(t) d t=-\int_{0}^{\omega}\left|x_{n}^{\prime \prime}(t)\right|^{2} d t$ into (4.10),

$$
\begin{aligned}
& \int_{0}^{\omega}\left|x_{n}^{\prime \prime}(t)\right|^{2} d t \\
& \quad=\int_{0}^{\omega} a(t) x_{n}(t) x_{n}^{\prime}(t) d t-\int_{0}^{\omega} f_{n}\left(t, x_{n}(t)+\gamma(t)\right) x_{n}^{\prime}(t) d t \\
& \quad \leq\|a\| \int_{0}^{\omega}\left|x_{n}(t)\right|\left|x_{n}^{\prime}(t)\right| d t+\int_{0}^{\omega}\left|f_{n}\left(t, x_{n}(t)+\gamma(t)\right)\right|\left|x_{n}^{\prime}(t)\right| d t \\
& \quad \leq\|a\| R \sqrt{\omega}\left(\int_{0}^{\omega}\left|x_{n}^{\prime}(t)\right|^{2} d t\right)^{\frac{1}{2}}+\left(\int_{0}^{\omega}\left|f_{n}\left(t, x_{n}(t)+\gamma(t)\right)\right|^{2} d t\right)^{\frac{1}{2}}\left(\int_{0}^{\omega}\left|x_{n}^{\prime}(t)\right|^{2} d t\right)^{\frac{1}{2}} \\
& \quad=\left(\|a\| R \sqrt{\omega}+\left\|f_{R}\right\|_{2}\right)\left(\int_{0}^{\omega}\left|x_{n}^{\prime}(t)\right|^{2} d t\right)^{\frac{1}{2}},
\end{aligned}
$$

where

$$
\left|f_{R}\right|=\max _{\vartheta \leq x_{n}(t)+\gamma \leq R+\gamma^{*}}\left|f_{n}\left(t, x_{n}(t)+\gamma(t)\right)\right|, \quad\left\|f_{R}\right\|_{2}=\left(\int_{0}^{\omega}\left|f_{R}\right|^{2} d t\right)^{\frac{1}{2}} .
$$

Using the Writinger inequality (see [17], Lemma 2.4), we have

$$
\int_{0}^{\omega}\left|x_{n}^{\prime \prime}(t)\right|^{2} d t \leq\left(\|a\| R \sqrt{\omega}+\left\|f_{R}\right\|_{2}+\frac{M}{n_{0}} \sqrt{\omega}\right)\left(\frac{\omega}{2 \pi}\right)\left(\int_{0}^{\omega}\left|x_{n}^{\prime \prime}(t)\right|^{2} d t\right)^{\frac{1}{2}} .
$$

It is easy to see that there exists a constant $D_{1}^{\prime}>0$ such that

$$
\int_{0}^{\omega}\left|x_{n}^{\prime \prime}(t)\right|^{2} d t \leq D_{1}^{\prime}
$$

From $x_{n}(0)=x_{n}(\omega)$, we know that there exists a point $t_{0} \in[0, \omega]$ such that $x_{n}^{\prime}\left(t_{0}\right)=0$. Therefore, we have

$$
\left\|x_{n}^{\prime}\right\|=\max _{t}\left|\int_{t_{0}}^{t} x_{n}^{\prime \prime}(s) d s\right| \leq \int_{0}^{\omega}\left|x_{n}^{\prime \prime}(s)\right| d s \leq \omega^{\frac{1}{2}}\left(\int_{0}^{\omega}\left|x_{n}^{\prime \prime}(s)\right|^{2} d s\right)^{\frac{1}{2}} \leq \omega^{\frac{1}{2}} D_{1}^{\prime \frac{1}{2}}:=D_{1} .
$$


The fact that $\left\|x_{n}\right\|<R$ and (4.8) show that $\left\{x_{n}\right\}_{n \in N_{0}}$ is bounded and an equi-continuous family on $R$. Now the Arzelà-Ascoli theorem guarantees that $\left\{x_{n}\right\}_{n \in N_{0}}$ has a subsequence $\left\{x_{n_{k}}\right\}_{k \in N}$, converging uniformly on $R$ to a function $x \in X$. From the fact that $\left\|x_{n}\right\|<R$ and $\vartheta \leq x_{n}+\gamma$, we know that $x$ satisfies $\vartheta \leq x(t)+\gamma(t) \leq R+\gamma^{*}$ for all $t$. Moreover, $x_{n_{k}}$ satisfies the integral equation

$$
x_{n_{k}}(t)=P\left(f\left(t, x_{n_{k}}(t)+\gamma(t)\right)+\frac{M}{n_{k}}\right) .
$$

Let $k \rightarrow \infty$, we get

$$
x(t)=P(f(t, x(t)+\gamma(t))) .
$$

Therefore, $x$ is a positive periodic solution of (4.2) and satisfies $0<\|x\| \leq R$. Besides, it is not difficult to show that $\|x\|<R$, by noting that if $\|x\|=R$, the argument similar to the proof of the first claim will yield a contradiction.

Combining the above three steps, the proof is completed.

Corollary 4.3 Assume that $\frac{3 \sqrt{3} \omega M^{\frac{1}{3}}}{4 \pi}<1$ holds. Suppose the following condition is satisfied:

$\left(F_{5}\right)$ there exist continuous functions $d(t), \hat{d}(t) \succ 0$ and $\rho>0,0 \leq \eta<1$ such that

$$
0 \leq \frac{\hat{d}(t)}{x^{\rho}} \leq f(t, x) \leq \frac{d(t)}{x^{\rho}}+d(t) x^{\eta} \quad \text { for all } x>0 \text { and a.e.t. }
$$

If $\gamma_{*} \geq 0$, then (1.5) has at least one positive $\omega$-periodic solution.

Proof We will apply Theorem 4.2. We take

$$
\phi_{L}(t)=\frac{\hat{d}(t)}{L^{\rho}}, \quad \zeta(t)=d(t), \quad g(x)=\frac{1}{x^{\rho}}, \quad h(x)=x^{\eta} .
$$

Then $\left(H_{1}\right)$ and $\left(H_{2}\right)$ are satisfied and the existence condition $\left(H_{6}\right)$ becomes

$$
\frac{m}{M}\left(\frac{R\left(\frac{\sigma m}{M} R+\gamma_{*}\right)^{\rho}}{1+\left(R+\gamma^{*}\right)^{\rho+\eta}}\right)>\Psi^{*}
$$

where $\Psi(t)=\int_{0}^{\omega} G(t, s) d(s) d s$, for some $r>0$. Since $\rho>0,0 \leq \eta<1$ and $\gamma_{*} \geq 0$, we can choose $R>0$ large enough that (4.11) is satisfied.

From Theorems 3.6 and 4.2, we obtain the following conclusion.

Theorem 4.4 Assume that $M<\frac{64 \pi^{3}}{81 \sqrt{3} \omega^{3}},\left(H_{1}\right)$ and $\left(H_{2}^{\prime}\right)$ hold. Suppose the following conditions are satisfied:

$\left(H_{6}^{\prime}\right)$ there exists a positive constant $R>0$ such that

$$
\frac{M}{m} g\left(\frac{\sigma m}{M} R+\gamma_{*}\right) \Lambda^{*}<R,
$$

and we assume that $f(t, x) \geq 0$ for all $(t, x) \in[t, \omega] \times(0, R]$. 
If $\gamma_{*} \geq 0$, then (1.5) has at least one positive $\omega$-periodic solution $x$ with $x(t)>\gamma(t)$ for all t and $0<\|x-\gamma\|<R$.

Corollary 4.5 Assume that $M<\frac{64 \pi^{3}}{81 \sqrt{3} \omega^{3}}$ holds and the nonlinearity in (1.5) is

$$
f(t, x)=\frac{1}{x^{\alpha}}-\frac{\kappa}{x^{\beta}}
$$

where $\alpha>\beta>0$ and $\mu>0$ is a positive parameter. If $\gamma_{*} \geq 0$, then there exists a positive constant $\kappa_{2}$ such that (1.5) has at least one positive periodic solution for each $0 \leq \kappa \leq \kappa_{2}$.

Proof We will apply Theorem 4.4. Take

$$
g(x)=\frac{1}{x^{\alpha}}, \quad \zeta(t) \equiv 1
$$

It is easy to see that the condition $\left(H_{2}^{\prime}\right)$ holds. The existence condition $\left(H_{6}^{\prime}\right)$ becomes

$$
m R\left(\frac{\sigma m}{M} R+\gamma_{*}\right)^{\alpha}>1
$$

where $\frac{1}{M}=\int_{0}^{\omega} G(t, s) d s$. we can choose $R>0$ appropriately large such that (4.12) is satisfied.

Next, we show that the nonlinear term $f(t, x) \geq 0$, for all $(t, x) \in[0, \omega] \times(0, R]$. In fact, $f(t, x) \geq 0$ if and only if $\kappa \leq x^{\beta-\alpha}$. There exists a positive constant $\kappa_{2}$ such that $\kappa_{2}<R^{\beta-\alpha}$. In view of $\kappa<\kappa_{2}$ and $\beta<\alpha$, we get $\kappa<R^{\beta-\alpha}<x^{\beta-\alpha}$ for all $x \in(0, R]$. Therefore, the condition $\left(H_{6}^{\prime}\right)$ holds.

Finally, we show that $\left(H_{1}\right)$ is satisfied. Let

$$
P(x)=\frac{1}{x^{\alpha}}-\frac{\kappa}{x^{\beta}}, \quad x \in(0,+\infty),
$$

and

$$
s_{1}=\kappa^{-\frac{1}{\alpha-\beta}}, \quad s_{2}=\left(\frac{\alpha}{\kappa \beta}\right)^{\frac{1}{\alpha-\beta}} .
$$

Since $\alpha>\beta$, one can easily verify that $s_{1}<s_{2}$ and $P\left(s_{1}\right)=0, P^{\prime}\left(s_{2}\right)=0, P^{\prime}(s)<0, s \in\left(0, s_{2}\right)$. Therefore, $P(s)$ is decreasing in $\left(0, s_{1}\right) \subset\left(0, s_{2}\right)$. On the other hand, we can choose $\kappa>0$ small enough such that $R \in\left(0, s_{1}\right)$. Thus,

$$
\min _{s \in(0, R)} P(s)=P(R)>P\left(s_{1}\right)=0 .
$$

This implies that the condition $\left(H_{1}\right)$ is satisfied if we take

$$
\phi_{R}(t) \equiv P(R)
$$

Theorem 4.6 Assume that $M<\frac{64 \pi^{3}}{81 \sqrt{3} \omega^{3}}$ and $\left(H_{2}\right)$ hold. Assume that the following condition holds: 
$\left(H_{6}^{*}\right)$ There exists a positive constant $R>0$ such that

$$
\frac{M}{m} g\left(\frac{\sigma m}{M} R\right)\left(1+\frac{h\left(R+\gamma^{*}\right)}{g\left(R+\gamma^{*}\right)}\right) \Lambda^{*}<R .
$$

If $\gamma_{*}>0$, then (1.5) has at least one positive $\omega$-periodic solution $x$ with $x(t)>\gamma(t)$ for all $t$ and $0<\|x-\gamma\|<R$.

Proof The proof of this theorem can be completed by the method analogous to that in Theorem 4.2. Step 1 and Step 2 are the same as in the proof of Theorem 4.2. Now, we consider that $x_{n}(t)+\gamma(t)$ have a uniform positive lower bound, i.e., there exists a constant $\vartheta_{1}>0$, independent of $n \in N_{0}$, such that

$$
\min _{t \in[0, \omega]}\left\{x_{n}(t)+\gamma(t)\right\} \geq \vartheta_{1}
$$

for all $n \in N_{0}$.

Since $x_{n}+\gamma>\frac{1}{n}$ and $\gamma_{*}>0$, from Lemma 2.2, we know that $G$ and $f$ are of a non-negative sign. Thus we have $x_{n}(t)+\gamma(t) \geq \gamma(t) \geq \gamma_{*} \geq \vartheta_{1}$. The rest of the proof is the same as Theorem 4.2.

Corollary 4.7 Assume that $M<\frac{64 \pi^{3}}{81 \sqrt{3} \omega^{3}}$ holds. Suppose the following condition is satisfied:

$\left(F_{6}\right)$ there exist continuous function $d(t) \geq 0$ for a.e. $t \in[0, \omega]$ and $\rho>0,0 \leq \eta<1$ such that

$$
0 \leq f(t, x) \leq \frac{d(t)}{x^{\rho}}+d(t) x^{\eta}, \quad \text { for all } x>0, \text { for a.e. } t
$$

If $\gamma_{*}>0$, then (1.5) has at least one positive $\omega$-periodic solution.

Proof We will apply Theorem 4.6. Take

$$
k(t)=d(t), \quad g(x)=\frac{1}{x^{\rho}}, \quad h(x)=x^{\eta},
$$

then $\left(H_{2}\right)$ is satisfied and the existence condition $\left(H_{6}^{*}\right)$ becomes

$$
\frac{m}{M}\left(\frac{R\left(\frac{\sigma m}{M} R+\gamma_{*}\right)^{\rho}}{1+\left(R+\gamma^{*}\right)^{\rho+\eta}}\right)>\Psi^{*}
$$

Since $\tau>0,0 \leq \eta<1$ and $\gamma_{*}>0$, we can choose $R>0$ large enough such that (4.13) is satisfied.

\subsection{Case (II) $\gamma^{*} \leq 0$}

Theorem 4.8 Assume that $M<\frac{64 \pi^{3}}{81 \sqrt{3} \omega^{3}},\left(H_{1}\right)$ and $\left(H_{2}\right)$ hold, and the following condition is satisfied:

$\left(H_{6}^{* *}\right)$ There exists a positive constant $R>0$ such that $\frac{\sigma m}{M} R+\gamma_{*}>0$ and

$$
\frac{M}{m} g\left(\frac{\sigma m}{M} R+\gamma_{*}\right)\left(1+\frac{h(R)}{g(R)}\right) \Lambda^{*}<R
$$


$\left(H_{7}\right) \gamma_{*}+\Phi_{*}^{\prime}>0$, here $\Phi^{\prime}(t)=\int_{0}^{\omega} G(t, s) \phi_{R}(s) d s$.

If $\gamma^{*} \leq 0$, then (1.5) has at least one positive $\omega$-periodic solution $x$ with $x(t)>\gamma(t)$ for all tand $0<\|x-\gamma\|<R$.

Proof The proof of this theorem can be completed by the method analogous to that in Theorem 4.2. Step 1 and Step 2 are the same as in the proof of Theorem 4.2. Now, it remains to consider that $x_{n}(t)+\gamma(t)$ has a uniform positive lower bound, i.e., there exists a constant $\vartheta_{2}>0$, independent of $n \in N_{0}$, such that

$$
\min _{t \in[0, \omega]}\left\{x_{n}(t)+\gamma(t)\right\} \geq \vartheta_{2}
$$

for all $n \in N_{0}$.

Since $\left(H_{1}\right)$ and $\gamma^{*} \leq 0$, we know that there exists a continuous function $\phi_{R}(t)>0$ such that $f(t, x) \geq \phi_{R}(t)$ for a.e. $t$ and $0<x \leq R+\gamma^{*} \leq R$. Let $x_{R}(t)$ be the unique $\omega$-periodic solution to

$$
x^{\prime \prime \prime}(t)+a(t) x(t)=\phi_{R}(t)
$$

from $\left(H_{6}^{*}\right)$, then we have

$$
\begin{aligned}
x_{R}(t)+\gamma(t) & =P\left(\phi_{R}(t)\right)+\gamma(t) \geq T\left(\phi_{R}(t)\right)+\gamma(t) \\
& =\int_{0}^{\omega} G(t, s) \phi_{R}(s) d s+\gamma(t) \\
& \geq \Phi_{*}^{\prime}+\gamma_{*}>0,
\end{aligned}
$$

Since $x_{n}(t)+\gamma(t) \leq R+\gamma^{*}<R$ and $x_{n}+\gamma_{*} \geq \frac{1}{n}$, we have

$$
\begin{aligned}
x_{n}(t)+\gamma(t) & =P\left(f_{n}\left(s, x_{n}(s)+\gamma(s)\right)+\frac{M}{n}\right)+\gamma(t) \\
& \geq T\left(f_{n}\left(s, x_{n}(s)+\gamma(s)\right)+\frac{M}{n}\right)+\gamma(t) \\
& =\int_{0}^{\omega} G(t, s) f_{n}\left(s, x_{n}(s)+\gamma(s)\right) d s+\gamma(t)+\frac{1}{n} \\
& \geq \int_{0}^{\omega} G(t, s) \phi_{R}(s) d s+\gamma(t) \\
& \geq \Phi_{*}^{\prime}+\gamma_{*}:=\vartheta_{2} .
\end{aligned}
$$

So we have $x_{n}(t)+\gamma(t) \geq \vartheta_{2}$. The rest of the proof is the same as Theorem 4.2.

Remark 2 Replacing above assumptions $M<\frac{64 \pi^{3}}{81 \sqrt{3} \omega^{3}}$ by assumption $M<\frac{8 \pi}{3 \sqrt{3} \omega^{3}}$, we can get similar existence results, which we omit here.

\section{Conclusions}

The paper is devoted to the existence of a positive periodic solution for Eq. (1.5). As is well known, it is very complicated to calculate the Green's function of the third-order 
linear differential equation with variable coefficients. In this paper, we first discuss the Green's function of the third-order linear differential equation with constant coefficients (1.6). By application of the Green's function of (1.6) and some fixed point theorems, i.e. Schauder's fixed point theorem and a nonlinear alternative principle of Leray-Schauder, we obtain the existence of a positive periodic solution for (1.5). Our results are applicable to the case of a strong singularity as well as to the case of a weak singularity; these new results generalize some recent results obtained in [8].

\section{Acknowledgements}

SWY and $J$ are grateful to anonymous referees for their constructive comments and suggestions which have greatly improved this paper.

Funding

This work was supported by National Natural Science Foundation of China under Grand [11626087, 11601048]; and Fundamental Research Funds for the Universities of Henan Province under Grand [NSFRF170302]; and Henan Polytechnic University Doctor Fund under Grand [B2016-058].

Availability of data and materials

Data sharing not applicable to this article as no data sets were generated or analyzed during the current study.

Competing interests

The authors declare that there is no conflict of interests regarding the publication of this article.

Authors' contributions

SWY and $J L$ contributed equally and significantly in writing this article. Both authors read and approved the final manuscript.

\section{Publisher's Note}

Springer Nature remains neutral with regard to jurisdictional claims in published maps and institutional affiliations.

Received: 14 June 2018 Accepted: 16 December 2018 Published online: 01 February 2019

\section{References}

1. Agarwal, R., O'Regan, D., Wong, R.: Positive Solutions of Differential, Difference and Integral Equations. Kluwer Academic Publisher, Dordrecht (1999)

2. Cheng, Z., Rem, J.: Studies on a damped differential equation with repulsive singularity. Math. Methods Appl. Sci. 36, 983-992 (2013)

3. Cheng, Z., Ren, J.: Periodic and subharmonic solutions for Duffing equation with singularity. Discrete Contin. Dyn. Syst., Ser. A 32, 1557-1574 (2012)

4. Cheng, Z., Ren, J.: Positive solutions for third-order variable-coefficient nonlinear equation with weak and strong singularities. J. Differ. Equ. Appl. 21, 1003-1020 (2015)

5. Cheng, Z., Ren, J.: Periodic solution for second order damped differential equations with attractive-repulsive singularities. Rocky Mt. J. Math. 48, 753-768 (2018)

6. Chu, J., Torres, P.: Applications of Schauder's fixed point theorem to singular differential equations. Bull. Lond. Math. Soc. 39, 653-660 (2007)

7. Chu, J., Torres, P., Zhang, M.: Periodic solution of second order non-autonomous singular dynamical systems. J. Differ Equ. 239, 196-212 (2007)

8. Chu, J., Zhou, Z.: Positive solutions for singular non-linear third-order periodic boundary value problems. Nonlinear Anal. TMA 64, 1528-1542 (2006)

9. Fonda, A., Manásevich, R., Zanolin, F.: Subharmonics solutions for some second order differential equations with singularities. SIAM J. Math. Anal. 24, 1294-1311 (1993)

10. Hakl, R., Torres, P.: On periodic solutions of second-order differential equations with attractive-repulsive singularities. J. Differ. Equ. 248, 111-126 (2010)

11. Lazer, A., Solimini, S.: On periodic solutions of nonlinear differential equations with singularities. Proc. Am. Math. Soc 99, 109-114 (1987)

12. Ma, R., Chen, R., He, Z.: Positive periodic solutions of second-order differential equations with weak singularities. Appl. Math. Comput. 232, 97-103 (2014)

13. Rachunková, I., Tvrdý, M., Vrkoć, l.: Existence of nonnegative and nonpositive solutions for second order periodic boundary value problems. J. Differ. Equ. 176, 445-469 (2001)

14. Rem, J., Siegmund, S., Chen, Y.: Positive periodic solutions for third-order nonlinear differential equations. Electron. J. Differential Equations 2011, 66 (2011)

15. Sun, J., Liu, Y.: Multiple positive solutions of singular third-order periodic boundary value problem. Acta Math. Sci. 25, 81-88 (2005)

16. Torres, P.: Weak singularities may help periodic solutions to exist. J. Differ. Equ. 232, 277-284 (2007)

17. Torres, P., Cheng, Z., Ren, J.: Non-degeneracy and uniqueness of periodic solutions for $2 \mathrm{n}$-order differential equation. Discrete Contin. Dyn. Syst., Ser. A 33, 2155-2168 (2013) 
18. Wang, H.: Positive periodic solutions of singular systems with a parameter. J. Differ. Equ. 249, 2986-3002 (2010)

19. Wang, Z: Periodic solutions of Liénard equation with a singularity and a deviating argument. Nonlinear Anal., Real World Appl. 16, 227-234 (2014)

20. Wang, Z., Ma, T.: Existence and multiplicity of periodic solutions of semilinear resonant Duffing equations with singularities. Nonlinearity 25, 279-307 (2012)

21. Zeidler, E.: Applied Functional Analysis. Applied Mathematical Sciences, vol. 108. Springer, Berlin (1995)

22. Zhang, M.: Periodic solutions of Liénard equations with singular forces of repulsive type. J. Math. Anal. Appl. 203, 254-269 (1996)

Submit your manuscript to a SpringerOpen ${ }^{\circ}$ journal and benefit from:

- Convenient online submission

- Rigorous peer review

- Open access: articles freely available online

- High visibility within the field

- Retaining the copyright to your article

Submit your next manuscript at $\gg$ springeropen.com 